\title{
Industrial Waste
}

National Cancer Institute

\section{Source}

National Cancer Institute. Industrial Waste. NCI Thesaurus. Code C577.

Materials that are discarded following industrial processes. 